\title{
Neuropathie motrice et expansion ancestrale dans le gène VWA1
}

\section{Enzo Cohen}

\section{Résumé}

La matrice extra-cellulaire (MEC) est un réseau de macromolécules telles que les collagènes, les protéoglycanes et les glycoprotéines. Le gène VWA1 (pour von Willebrand factor A domain containing 1) code un composant de la MEC interagissant avec le perlecan et le collagène VI. Les souris invalidées pour le gène Vwa1 présentent des anomalies de la fonction et de la structure du nerf périphérique ; cependant, aucune mutation de ce gène n'a encore été impliquée en pathologie humaine. En criblant les génomes des 74180 individus issus du projet anglais $100 \mathrm{~K}$ Genomes puis par séquençage ciblé, les auteurs ont identifié quinze familles présentant une forme autosomique récessive de neuropathie motrice héréditaire, porteurs de variations bi-alléliques rares dans le gène VWA1 [1]. L'allèle causal p.(G25Rfs*74) est à l'origine d'une expansion de séquence répétée de 10 paires de bases et est présent dans quatorze des quinze familles étudiées. Des études d'haplotypage ayant mis en évidence une région partagée de $220 \mathrm{~kb}$ suggèrent que cette mutation fondatrice serait apparue dans la population européenne il y a plus de 7000 ans. Chez ces patients, les études histologiques et électrophysiologiques, ainsi que l'IRM musculaire, ont objectivé une atteinte du nerf périphérique avec quelques lésions musculaires sans doute secondaires au processus de dénervation. Par RT-PCR et western-blot, les auteurs ont montré que l'allèle ancestral entrainait la dégradation partielle de l'ARN messager par " nonsense-mediated decay " et conduisait, par voie de conséquence, à l'absence de protéine. Enfin, des modèles poisson-zèbre invalidés pour vwa1 présentent une diminution de la croissance axonale des motoneurones, des formations synaptiques des muscles squelettiques et des troubles de la locomotion. Les mutations bi-alléliques de VWA1 chez l'homme pourraient, dans la population européenne, être responsables d'environ $1 \%$ des neuropathies motrices héréditaires non étiquetées. L'implication de ce nouveau gène a vraisemblablement échappé à de précédentes analyses du fait de son fort pourcentage en GC et des difficultés computationnelles associées à la détection des régions répétées. La ré-analyse de données d'exomes précédemment non résolus pourrait mener à plus de diagnostics de ce genre à condition d'utiliser un seuillage de qualité plus permissif.

\section{Commentaire}

Cette étude, tout comme celle publiée simultanément dans le même journal par un consortium allemand [2], fait état de l'implication du gène VWA1 dans des formes autosomiques récessives de neuropathie motrice héréditaire, et permet ainsi d'établir un tableau clinique très précis de cette nouvelle entité. Se manifestant généralement précocement, cette maladie se caractérise par une atteinte déficitaire proximale et distale, prédominant dans les membres inférieurs, et souvent une malformation des pieds et des rétractions des tendons d'Achille. Comme pour les mutations des gènes codant le collagène VI, responsables de dystrophies musculaires congénitales rétractiles, les mutations de VWA1 soulignent ainsi l'importance de la matrice extracellulaire dans le maintien de la réactivité mécanique et la signalisation des fibres musculaires.

Cette étude confirme la puissance de l'exploitation de larges bases de données de séquençage à l'ère du " big data", ainsi que l'importance des collaborations internationales destinées à colliger un maximum de cas porteurs de variants dans un même gène et/ou partageant un phénotype similaire. Les auteurs soulignent les problèmes techniques associés au séquençage d'exome pouvant retarder l'identification de nouveaux gènes. Ces "angles morts" sont toutefois amenés à disparaitre avec la généralisation, en clinique, du séquençage de type " wholegenome". C'est le cas ici avec la mutation fondatrice p.(G25Rfs*74), caractérisée comme telle en raison du nombre anormalement élevé d'homozygotes observés et compte tenu de sa fréquence allélique d'environ $0,1 \%$ dans la population européenne. Sans exclure l'hypothèse d'un effet fondateur, l'étude allemande a identifié la duplication de cette région répétée ainsi que sa délétion dans d'autres populations, suggérant également son apparition de novo et soulignant l'instabilité mutationnelle du locus considéré [2]. D'autres variants tronquants ont été identifiés par les deux équipes citées mais seule la duplication mentionnée dans le premier article a fait l'objet d'évaluations fonctionnelles prouvant une perte de fonction. Il serait sans doute intéressant de démontrer la pathogénicité des variations faux-sens mises en évidence par les auteurs de ce dernier. La caractérisation de ces mutations pourrait non seulement contribuer à établir de nouveaux diagnostics pour cette maladie mais 
également permettre de déterminer des corrélations génotype/phénotype. Ce d'autant que les patients porteurs d'au moins un autre allèle que l'allèle ancestral décrit ci-dessus semblent présenter un phénotype plus modéré.

Motor neuropathy and founder expansion in the VWA1 gene

\section{LIENS D'INTÉRÊT}

L'auteur déclare n'avoir aucun lien d'intérêt concernant les données publiées dans cet article.

\section{RÉFÉRENCES}

1. Pagnamenta AT, Kaiyrzhanov R, Zou Y, et al. An ancestral 10-bp repeat expansion in VWA1 causes recessive hereditary motor neuropathy. Brain 2021; 3; $144: 584-600$

2. Deschauer M, Hengel H, Rupprich K, et al. Bi-allelic truncating mutations in VWA1 cause neuromyopathy. Brain $2021 ; 144$ : $574-83$

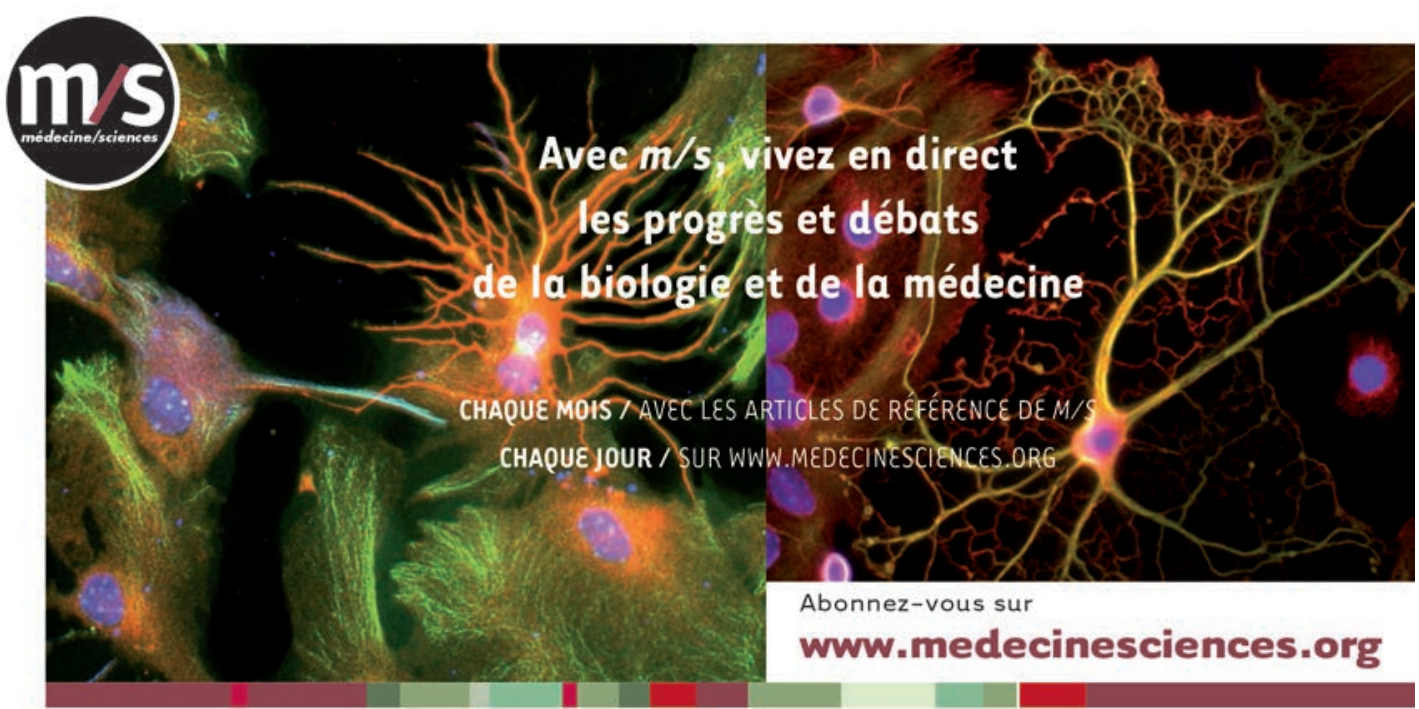

